\title{
Crosstalk Models for Short VDSL2 Lines from Measured 30 MHz Data
}

\author{
E. Karipidis, ${ }^{1}$ N. Sidiropoulos, ${ }^{1}$ A. Leshem, ${ }^{2}$ Li Youming, ${ }^{2}$ R. Tarafi, ${ }^{3}$ and M. Ouzzif ${ }^{3}$ \\ ${ }^{1}$ Department of Electronic and Computer Engineering, Technical University of Crete, 73100 Chania, Crete, Greece \\ ${ }^{2}$ Department of Electrical Engineering, Bar-Ilan University, 52900 Ramat-Gan, Israel \\ ${ }^{3}$ France Telecom R\&D, 22307 Lannion, France
}

Received 30 November 2004; Revised 25 April 2005; Accepted 2 August 2005

\begin{abstract}
In recent years, there has been a growing interest in hybrid fiber-copper access solutions, as in fiber to the basement (FTTB) and fiber to the curb/cabinet (FTTC). The twisted pair segment in these architectures is in the range of a few hundred meters, thus supporting transmission over tens of $\mathrm{MHz}$. This paper provides crosstalk models derived from measured data for quad cable, lengths between 75 and 590 meters, and frequencies up to $30 \mathrm{MHz}$. The results indicate that the log-normal statistical model (with a simple parametric law for the frequency-dependent mean) fits well up to $30 \mathrm{MHz}$ for both FEXT and NEXT. This extends earlier log-normal statistical modeling and validation results for NEXT over bandwidths in the order of a few MHz. The fitted crosstalk power spectra are useful for modem design and simulation. Insertion loss, phase, and impulse response duration characteristics of the direct channels are also provided.
\end{abstract}

Copyright $\odot 2006$ Hindawi Publishing Corporation. All rights reserved.

\section{INTRODUCTION}

Hybrid fiber-copper access solutions, such as fiber to the basement (FTTB) and fiber to the curb/cabinet (FTTC), entail twisted pair segments in the order of a few hundred meters-thus supporting transmission over up to $30 \mathrm{MHz}$. Very-high bit-rate digital subscriber line (VDSL) and the emerging VDSL2 draft are the pertinent high-speed transmission modalities for these lengths. This scenario is very different from the typical asymmetric digital subscriber line (ADSL) or high bit-rate digital subscriber line (HDSL) environment. For the shortest loops, for example, the shape of the far-end crosstalk (FEXT) power spectrum can be expected to be similar to the shape of the near-end crosstalk (NEXT) power spectrum; while it is a priori unclear that NEXT and FEXT models [3, 4] developed and fitted to ADSL/HDSL bandwidths, will hold up over a much wider bandwidth.

This paper describes the results of an extensive channel measurement campaign conducted by France Telecom R\&D, and associated data analysis undertaken by the authors in order to better understand the properties of these very short copper channels. A large number of FEXT, NEXT, and insertion loss (IL) channels were measured and analyzed, for lengths ranging from 75 to 590 meters and bandwidth up to $30 \mathrm{MHz}$. The main contribution is three-fold. First, the simple parametric models in [3] are tested and validated over the target lengths and range of frequencies. Second, the log-normal model for the marginal distribution of both NEXT and FEXT is validated, extending earlier results $[3,4]$. Finally certain key fitted model parameters are provided, which are important for system development and service provisioning.

The rest of this paper is structured as follows. Section 2 provides a concise description of the measurement process and associated apparatus, while Section 3 reviews the basic parametric models for IL, NEXT, and FEXT. Section 4 presents the main results: fitted models for the crosstalk spectra plus model validation (Sections 4.1, 4.2). Section 4 also provides useful data regarding IL (Section 4.3), and the phase and essential duration of the direct channels (Sections 4.4, 4.5). Conclusions are drawn in Section 5.

\section{DESCRIPTION OF THE CHANNEL MEASUREMENT PROCESS AND APPARATUS}

IL, NEXT, and FEXT were measured for different lengths of $0.4 \mathrm{~mm}$ gauge $\mathrm{S} 88.28 .4$ cable, comprising 14 quads $(14 \times 2=$ 28 loops) [7]. The measured lengths were 75, 150, 300, and 590 meters. A network analyzer (NA) was employed in the measurement process. A power splitter was used to inject half of the source power to the cable, while the other half was diverted to the reference input $\mathrm{R}$ of the NA. The output of the measured channel was connected to input A of the NA, 
and the ratio $\mathrm{A} / \mathrm{R}$ was recorded. When measuring crosstalk between pairs $i$ and $j$, pairs $i$ and $j$ were terminated using $120 \mathrm{ohm}$ resistances; all other pairs in the binder were left open-circuit.

An impedance transformer (balun) was used to connect the measured pair with the measurement device. The reference for the baluns is North Hills $0302 B B(10 \mathrm{kHz}-60 \mathrm{MHz})$, except for FEXT and IL for 300 and 590 meters, for which the reference is North Hills $413 B F(100 \mathrm{kHz}-100 \mathrm{MHz})$. Prior to taking actual measurements, a calibration procedure was employed to offset the combined effect of the baluns and the coaxial cables.

Three different network analyzers were used, depending on cable length.

(i) 75 meters. HP8753ES, resolution bandwidth $=20 \mathrm{~Hz}$.

(ii) 150 meters. $H P 8751 A$, resolution bandwidth $=20 \mathrm{~Hz}$.

(iii) 300 and 590 meters. HP4395A, resolution bandwidth $=100 \mathrm{~Hz}$.

For all the measurements, the setup was as follows.

(i) Source power $=15 \mathrm{dBm}$.

(ii) Start frequency $=10 \mathrm{kHz}$.

(iii) Stop frequency $=30 \mathrm{MHz}$.

(iv) Number of points $=801$.

(v) Frequency sweep scale $=$ logarithmic.

Fifteen $\mathrm{dBm}$ was the maximum source power available in the lab. For each measured length, all possible (i.e., $\left(\begin{array}{c}28 \\ 2\end{array}\right)=378$ ) crosstalk channels in the binder were actually measured. In addition to NEXT and FEXT, IL and phase for the 28 direct channels were also measured.

Due to the fact that measurements were taken in logarithmic frequency scale, there was a need to interpolate the measured data over a linear frequency scale. For each measured channel, shape-preserving piecewise cubic (Hermite) interpolation of the log-scale amplitude of the frequency samples was used, to obtain 6955 equispaced frequency samples (spacing $=4.3125 \mathrm{kHz}$ ) from the 801 measured log-scale frequency samples. The choice of frequency sweep scale (linear versus logarithmic) hinges on a number of factors. A logarithmic scale packs higher sample density in the lower frequencies, wherein NEXT and FEXT typically exhibit faster variation with frequency, and can be relatively close to the measurement error floor. In this case, a logarithmic frequency sweep naturally yields more reliable interpolated channel estimates in the lower frequencies. On the other hand, this comes at the expense of lower sample density in the higher frequencies.

\section{MODELING OF COPPER CHANNELS}

A good overview of twisted pair channel models can be found in [3] (see also [4-6]). A summary of the most pertinent facts follows.

\subsection{Insertion loss}

The magnitude squared of insertion loss obeys a simple parametric model [3]

$$
\left|H^{\mathrm{IL}}(f, l)\right|^{2}=\mathrm{e}^{-2 \alpha l \sqrt{f}},
$$

where $f$ is the frequency in $\mathrm{Hz}, l$ is the length of the channel, and $\alpha$ is a constant. In $\mathrm{dB}$,

$$
20 \log _{10}\left|H^{\mathrm{IL}}(f, l)\right|=\beta(l) \sqrt{f},
$$

where we have defined $\beta(l)=-20 \alpha l \log _{10}(\mathrm{e})$.

\subsection{NEXT}

NEXT can be modeled as $[3,4]$

$$
\left|H^{\mathrm{N}}(f)\right|^{2}=K f^{3 / 2},
$$

where $K$ is a log-normal random variable. In $\mathrm{dB}$,

$$
20 \log _{10}\left|H^{N}(f)\right|=10 \log _{10}(K)+15 \log _{10}(f),
$$

where now $10 \log _{10}(K)$ is a normal random variable. It follows that $20 \log _{10}\left|H^{N}(f)\right|$ is a normal variable, with frequency-dependent mean.

Lin [6] has shown that $10 \log _{10}(K)$ can be better modeled by a gamma distribution, under certain conditions. In particular, a gamma distribution can better fit the tails of the empirical distribution. On the other hand, the normal distribution is simpler and widely used in this context, because it fits quite well.

\subsection{FEXT}

FEXT can be modeled as [3]

$$
\left|H^{\mathrm{F}}(f, l)\right|^{2}=K(l) f^{2}\left|H^{\mathrm{IL}}(f, l)\right|^{2},
$$

where $K(l)$ is a log-normal random variable, which now depends on length, $l$. In $\mathrm{dB}$ and using (2),

$$
\begin{aligned}
20 \log _{10}\left|H^{F}(f, l)\right|= & 10 \log _{10}(K(l))+\beta(l) \sqrt{f} \\
& +20 \log _{10}(f)
\end{aligned}
$$

where now $10 \log _{10}(K(l))$ is a normal random variable, and thus $20 \log _{10}\left|H^{F}(f, l)\right|$ is a normal variable too, with frequency-dependent mean.

\section{RESULTS}

\subsection{Fitted cross-spectra and log-normal model validation}

Results for NEXT are presented first; FEXT follows, in order of increasing loop length. The NEXT power spectrum is approximately independent of loop length for the lengths considered, ${ }^{1}$ as can be verified from the fitted parameter in

\footnotetext{
${ }^{1}$ NEXT generally depends on loop length, see [1].
} 


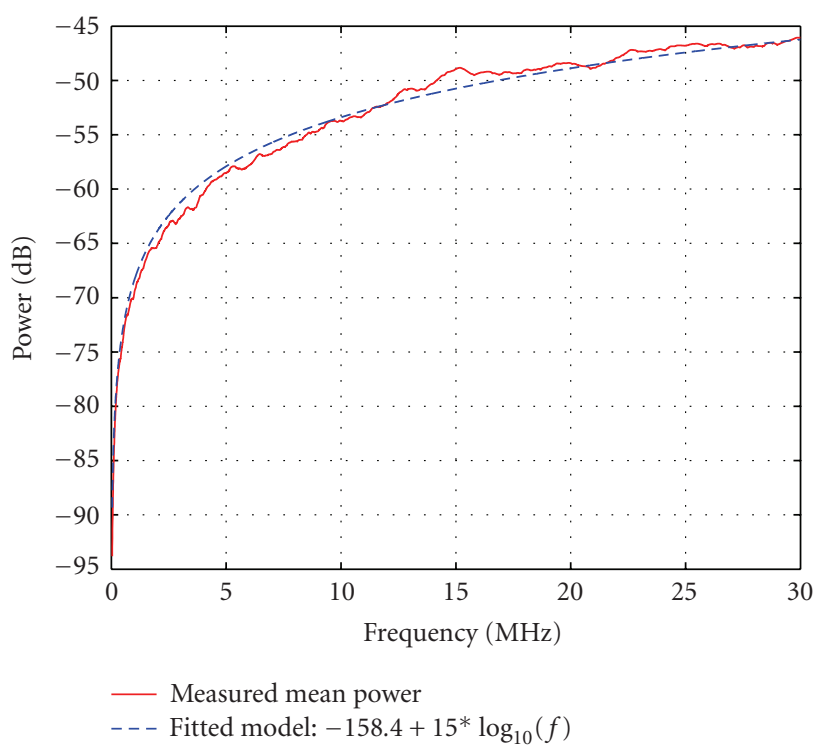

FIGURE 1: Measured mean power and fitted model for NEXT, $300 \mathrm{~m}$ $($ mean std $=9.5 \mathrm{~dB})$.

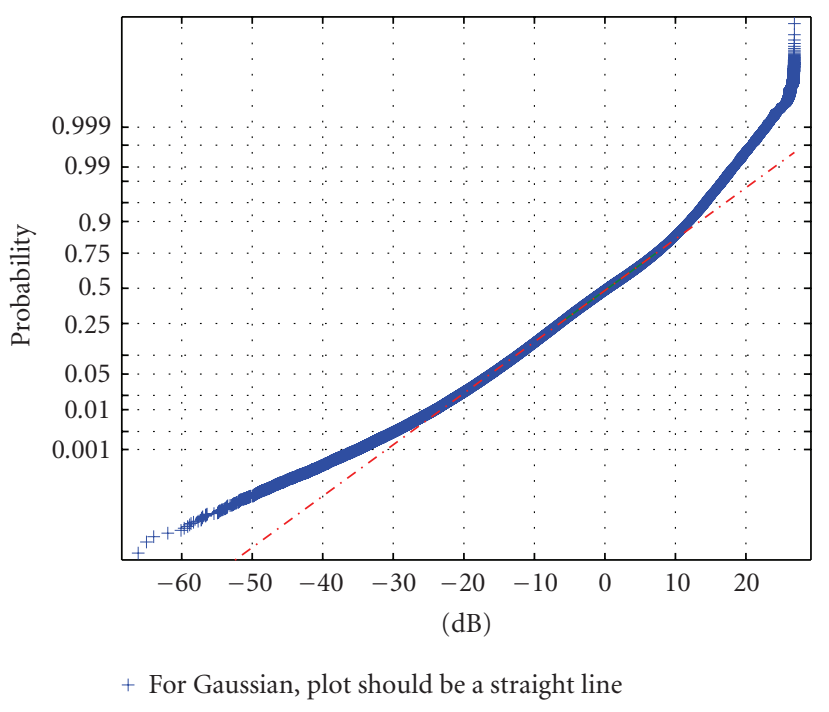

FIgURe 2: Deviation from Gaussian pdf for NEXT, $300 \mathrm{~m}$.

Figure 12. For brevity, detailed plots are therefore only provided for 300 meter NEXT. There are two plots per channel type and length considered. The first shows the measured mean log-power of all available channels of the given type, and the associated fitted model, as a function of frequency. As per Section 3, we use the following parametric model for the mean NEXT log-power:

$$
\mathrm{E}\left[20 \log _{10}\left|H^{N}(f)\right|\right] \approx c_{1}+15 \log _{10}(f),
$$

where $c_{1}=\mathrm{E}\left[10 \log _{10}(K)\right]$. The parameter $c_{1}$ is fitted to the model as follows. First, $\mathrm{E}\left[20 \log _{10}\left|H^{N}(f)\right|\right]$ is replaced by its sample estimate, $\mu_{\mathrm{s}}(f)$. Then, the sought parameter is fitted to $\mu_{\mathrm{s}}(f)$ in a least-squares (LS) sense. That is, $c_{1}$ is chosen to

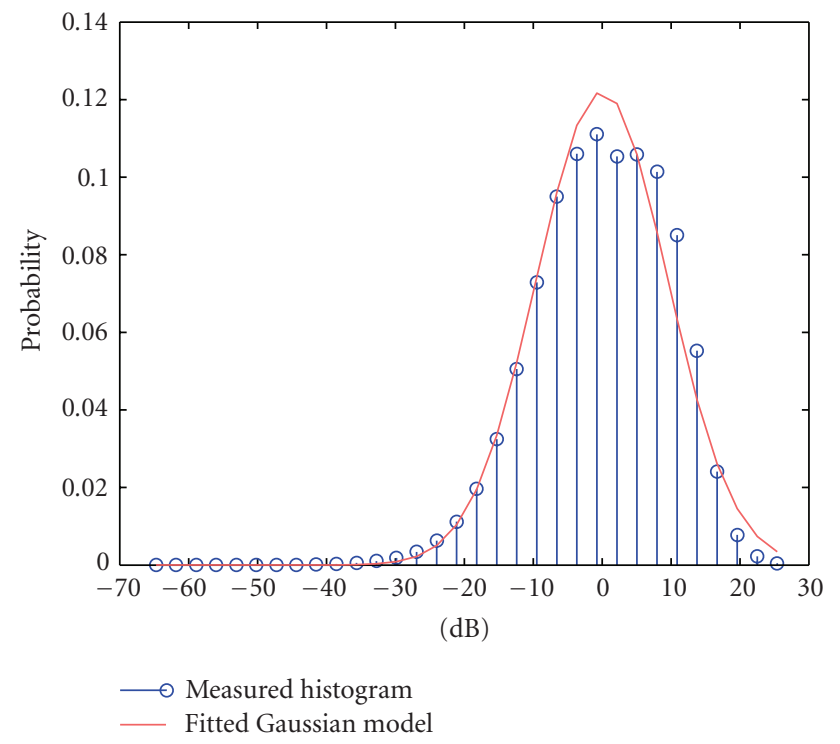

FIGURE 3: Histogram of the mean-centered power for NEXT, $300 \mathrm{~m}$.

minimize

$$
\sum_{f}\left|\mu_{s}(f)-\left(c_{1}+15 \log _{10}(f)\right)\right|^{2},
$$

yielding $\hat{c}_{1}$ equal to the mean of $\mu_{\mathrm{s}}(f)-15 \log _{10}(f)$. The situation is similar for FEXT, except that this time the parametric mean regression model is

$$
\mathrm{E}\left[20 \log _{10}\left|H^{F}(f, l)\right|\right] \approx c_{1}(l)+c_{2}(l) \sqrt{f}+20 \log _{10}(f),
$$

where $c_{1}(l)=\mathrm{E}\left[10 \log _{10}(K(l))\right]$ is now length-dependent, and $c_{2}(l) \equiv \beta(l)$, as per the associated discussion in Section 3 . Fitting the two parameters is a standard linear LS problem.

The fitted curve is plotted along with $\mu_{\mathrm{s}}(f)$ in the first of each pair of plots corresponding to each type of channel. The standard deviation (std) of the channel's log-power response is found to be approximately constant over the entire $30 \mathrm{MHz}$ frequency band; its average value is reported in the caption of the respective mean power plot.

After frequency-dependent mean removal ("centering" or "detrending") using the fitted parametric model, the residual frequency samples should behave like zero-mean normal random variables, if the log-normal model of the marginal distribution is correct. In the second plot of each pair, the validity of this assumption is assessed, by a socalled normal probability plot, which is produced using Matlab's normplot routine. The purpose of a normal probability plot is to graphically assess whether the data could come from a normal distribution. If so, the normal probability plot should be linear. Other distributions will introduce curvature in the plot. The normal probability plot helps in assessing deviations from normality, especially in the tails of the distribution. For $300 \mathrm{~m} \mathrm{NEXT}$, a third figure has been included showing a histogram of the mean-centered log-power responses, accumulated across all channels of the given type 


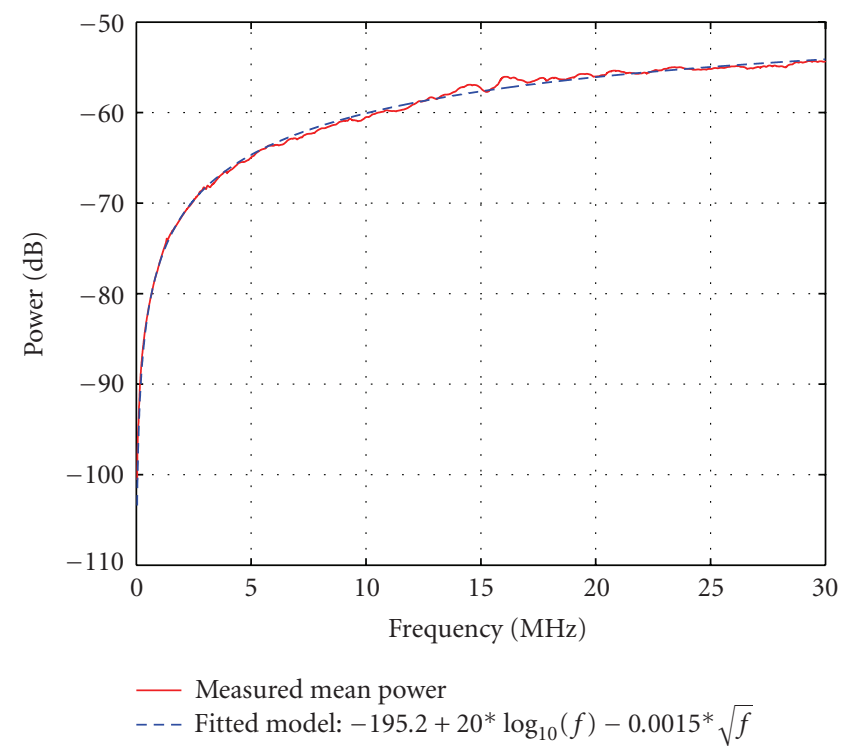

FIGURE 4: Measured mean power and fitted model for FEXT, $75 \mathrm{~m}$ (mean std $=9 \mathrm{~dB})$.

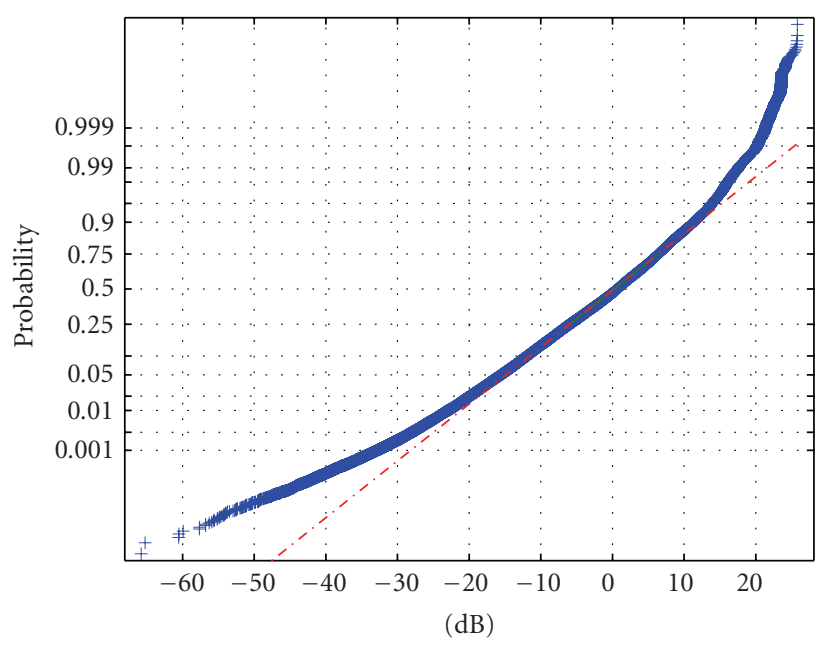

+ For Gaussian, plot should be a straight line

Figure 5: Deviation from Gaussian pdf for FEXT, 75 m.

and across all frequencies. A Gaussian probability density function has been fitted to the said data (not the histogram per se), and overlaid on top of the same plot. Gaussian fitting is performed in the maximum likelihood (ML) sense, which boils down to using the sample estimate of the variance of the centered data. This figure helps to assess (deviation from) normality, however tail inconsistencies are relatively hard to detect this way. For this reason, and for the sake of brevity, we are only showing normal probability plots for the FEXT channels.

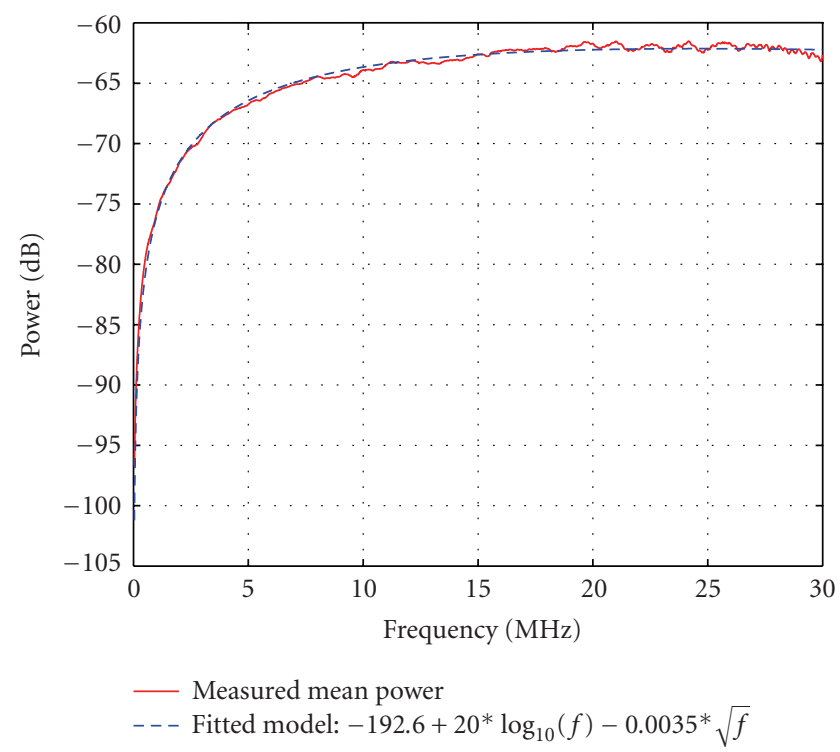

Figure 6: Measured mean power and fitted model for FEXT, $150 \mathrm{~m}$ $($ mean std $=9 \mathrm{~dB})$.

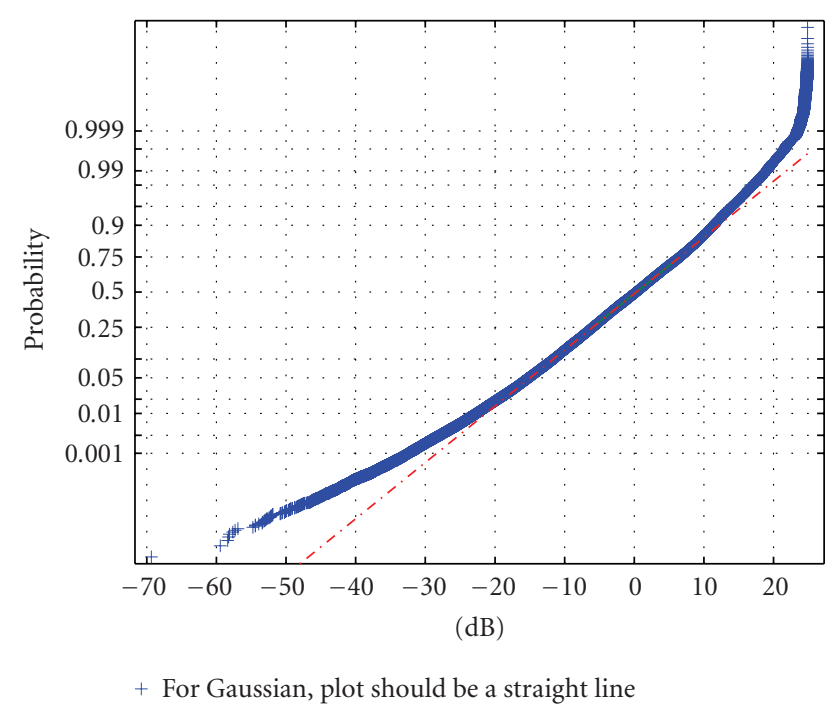

FIgUre 7: Deviation from Gaussian pdf for FEXT, $150 \mathrm{~m}$.

NEXT plots for 300 meters are presented in Figures 1, 2, and 3 . Figure 2 indicates that the normal distribution is a reasonable approximation, while a gamma distribution could be used to further improve the fit of the tails [6]. Plots for FEXT are shown in Figure pairs 4-5, 6-7, 8-9, and 10-11, for 75, 150,300 , and 590 meters, respectively.

The results indicate that the simple parametric models in [3] describe sufficiently well the mean log-power of the crosstalk channels, except for the 590 m FEXT case, where there is a noticeable deviation of the fitted model from the 


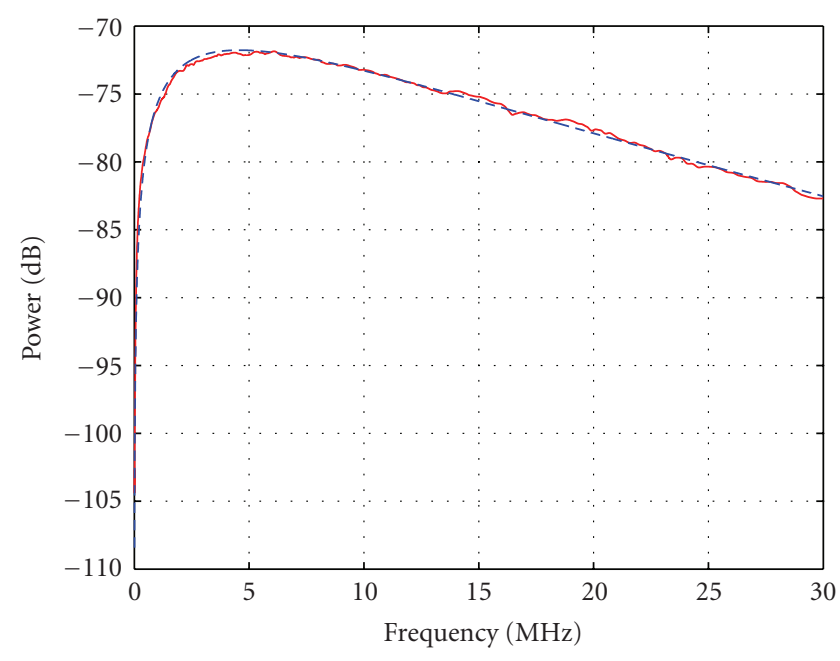

- Measured mean power
- - Fitted model: $-187.6+20 * \log _{10}(f)-0.0081 * \sqrt{f}$

FIGURE 8: Measured mean power and fitted model for FEXT, $300 \mathrm{~m}$ $($ mean std $=8.8 \mathrm{~dB})$.

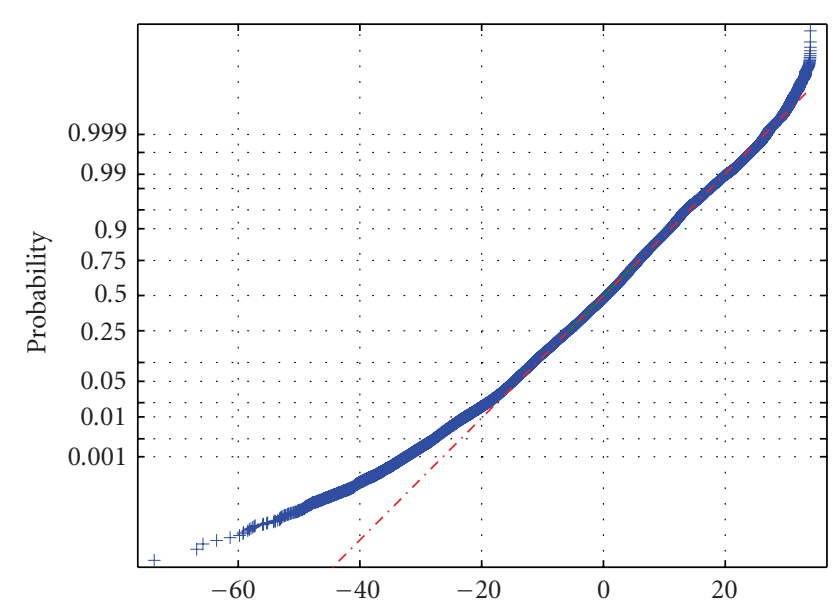

$(\mathrm{dB})$

+ For Gaussian, plot should be a straight line

Figure 9: Deviation from Gaussian pdf for FEXT, 300 m.

measured mean power, as high as $3 \mathrm{~dB}$ in the frequencies approximately up to $2 \mathrm{MHz}$ (see Figure 10). In order to obtain a better fit, we can generalize the model of (5) by relaxing the $f^{2}$ term to $f^{\gamma(l)}$, where $\gamma(l)$ is a length-dependent parameter. Then, (6) becomes

$$
\begin{aligned}
20 \log _{10}\left|H^{F}(f, l)\right|= & 10 \log _{10}(K(l))+\beta(l) \sqrt{f} \\
& +10 \gamma(l) \log _{10}(f)
\end{aligned}
$$

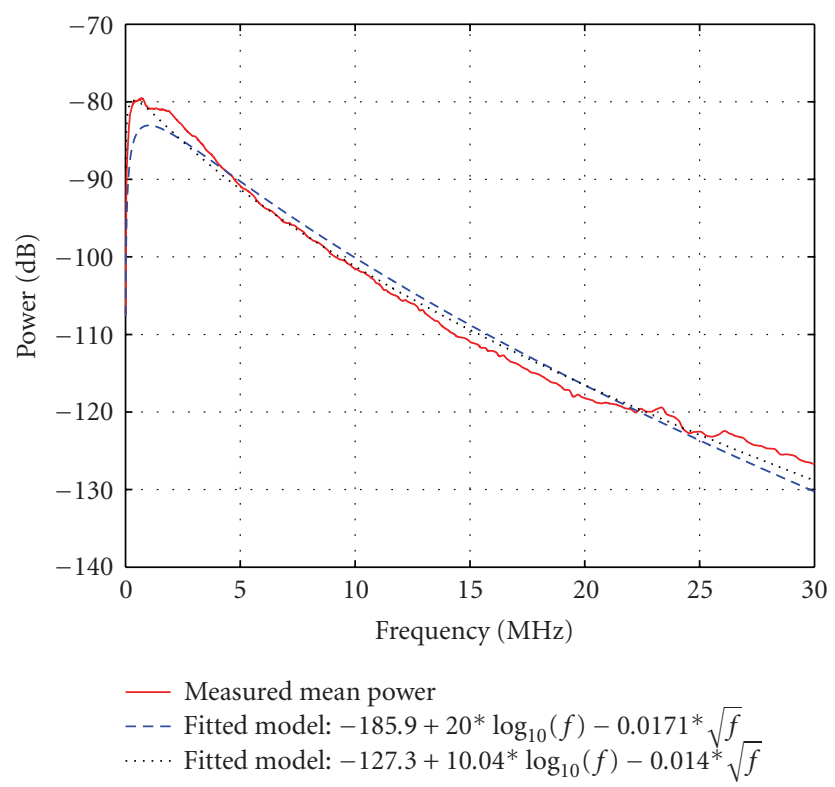

Figure 10: Measured mean power and fitted model for FEXT, $590 \mathrm{~m}$ $($ mean std $=11.2 \mathrm{~dB})$.

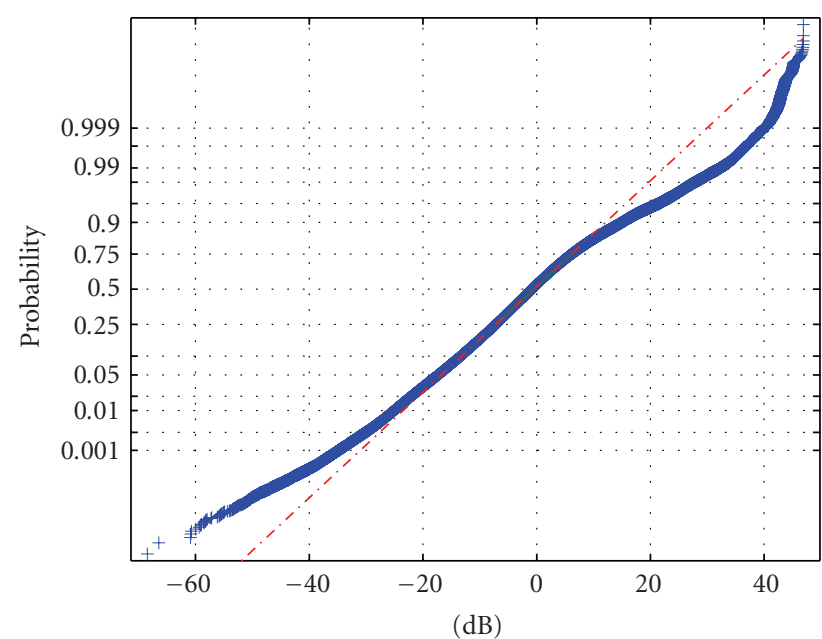

+ For Gaussian, plot should be a straight line

FIgure 11: Deviation from Gaussian pdf for FEXT, 590 m.

and the parametric mean regression model becomes

$$
\mathrm{E}\left[20 \log _{10}\left|H^{F}(f, l)\right|\right] \approx c_{1}(l)+c_{2}(l) \sqrt{f}+c_{3}(l) \log _{10}(f),
$$

where $c_{3}(l) \equiv 10 \gamma(l)$. That is, we are effectively introducing a third degree of freedom. The resulting profile and parameters of this fit are reported along with the original ones in Figure 10 for comparison purposes. 


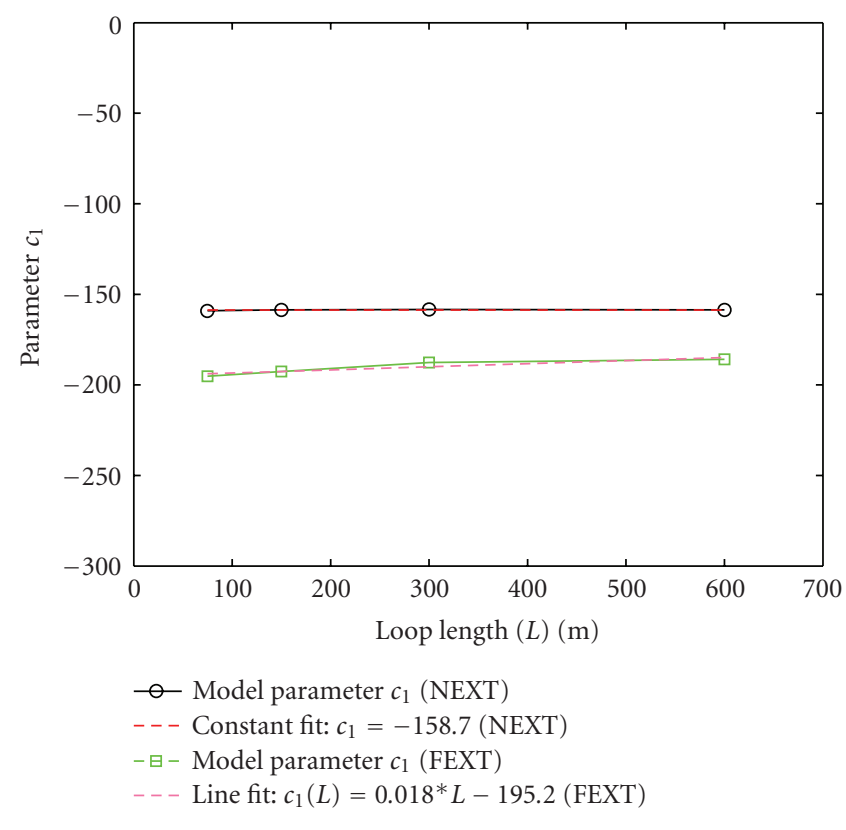

FIGURE 12: Fitted regression parameter $c_{1}$.

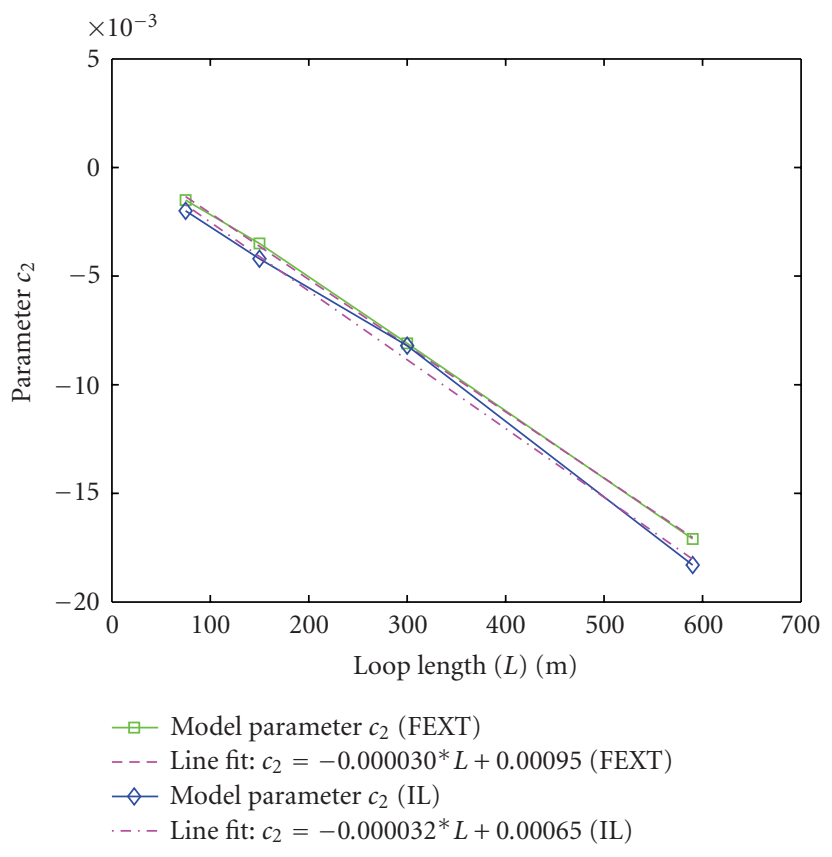

FIGURE 13: Fitted regression parameter $c_{2}$.

\subsection{Fitted regression parameters versus length}

The fitted frequency-dependent mean model parameters are also plotted in Figures 12 and 13, versus length. For NEXT, $c_{1} \approx-158.7$ ( -165.4 for Kerpez's model [4]) independent of length, as expected. For FEXT, both parameters show a nice

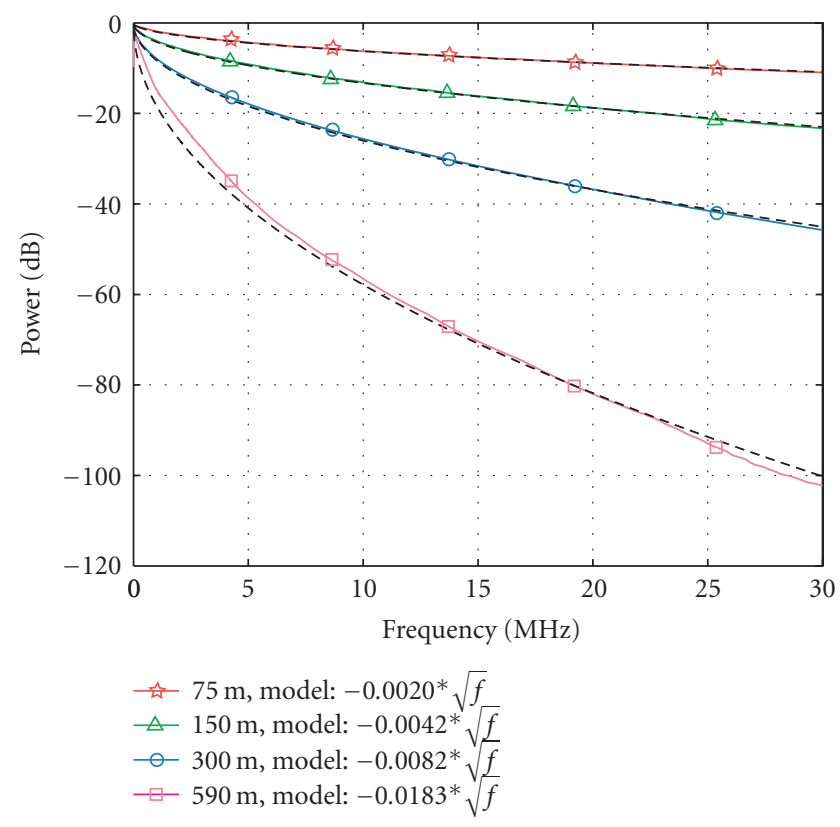

Figure 14: Measured mean power of direct channel and fitted model. (Insertion loss.)

affine dependence on length. In Figure 13 the fitted parameter $c_{2}(l) \equiv \beta(l)$ of the frequency-dependent mean model for the direct channel is shown to be an affine function of length as well.

\subsection{Insertion loss}

Figure 14 shows the sample mean IL (in $\mathrm{dB}$ ) and the associated fitted model, for all four lengths. Notice that the usable bandwidth indeed extends to $30 \mathrm{MHz}$ for the shortest (75 m) loop, but is effectively limited to about $7.5 \mathrm{MHz}$ for the longest ( $590 \mathrm{~m}$ ) loop considered. At that point, the loop's IL drops under $-50 \mathrm{~dB}$. Figure 13 shows the dependence on loop length of the model parameter $c_{2}(l) \equiv \beta(l)$ in (2).

\subsection{Phase of direct channels}

Figure 15 shows the unwrapped phase of all 28 direct channels, for 75, 150, 300, and 590 meters. Note that the (unwrapped) phase is approximately linear.

\subsection{Impulse response duration}

One parameter that is important from the viewpoint of modem design is the duration of the impulse response of the direct channel. For a multicarrier line code, this affects both the length of the cyclic prefix, and the number of taps (and thus cost and complexity) of the time-domain channel shortening equalizer (TEQ). We plot the $\mathrm{dB}$ magnitude of the direct channel's impulse response in Figures 16 and 17, for length 75 and 150 meters, respectively. The $99 \%$ energy breakpoint (the "essential duration" that contains $99 \%$ 


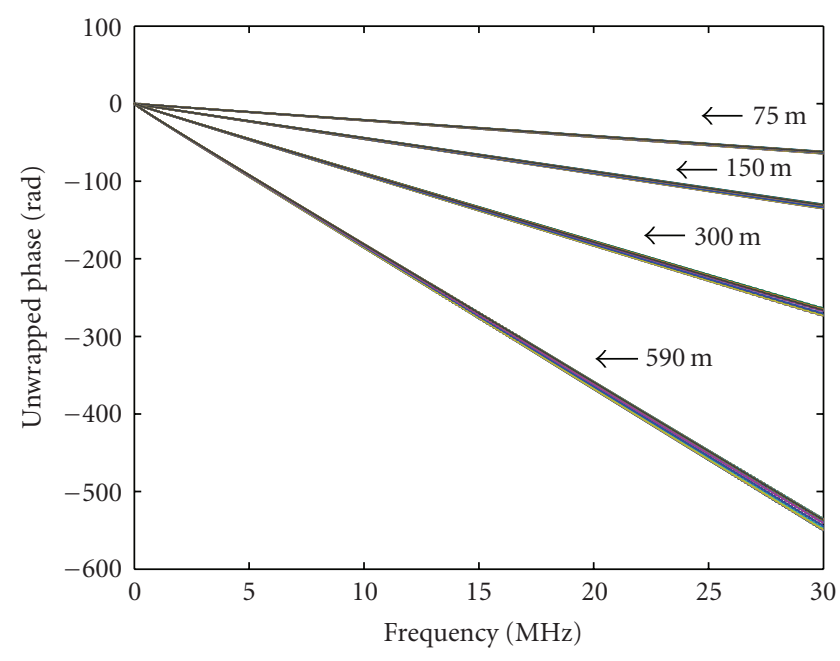

FIGURE 15: Unwrapped phase of all direct channels.

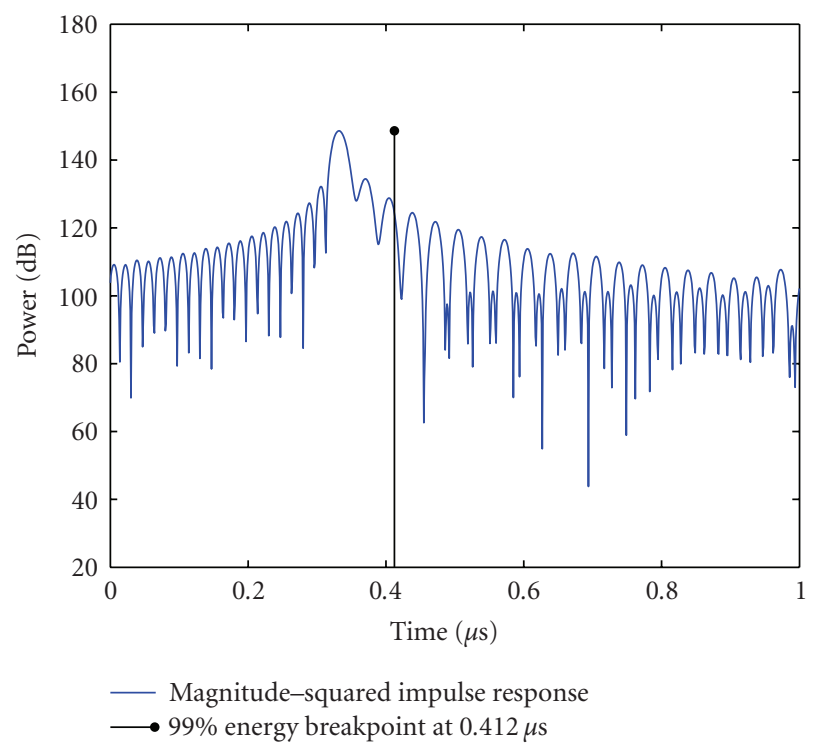

FIgURE 16: Magnitude-squared of direct channel's impulse response, $75 \mathrm{~m}$.

of the total energy) is also shown on each figure. The impulse responses were calculated via Riemann sum approximation ${ }^{2}$ of the inverse continuous-time Fourier transform of the interpolated frequency samples, using conjugate folding for the negative frequencies. Note that this approximation introduces aliasing error in the tails of the estimated impulse response. This is unavoidable, because we work with samples of

\footnotetext{
${ }^{2}$ For computational savings, this can be implemented via the (inverse) FFT.
}

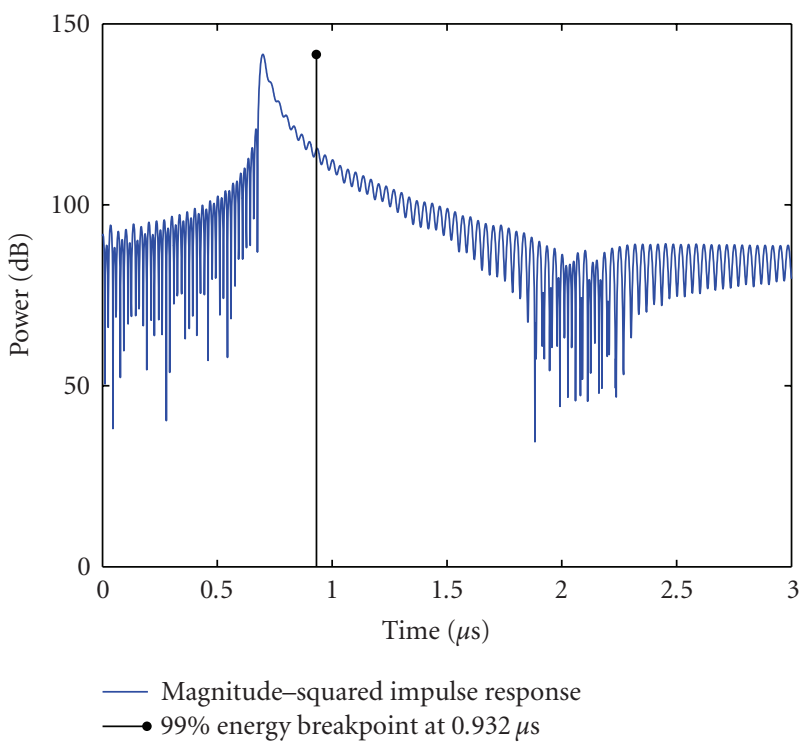

Figure 17: Magnitude-squared of direct channel's impulse response, $150 \mathrm{~m}$.

the continuous-time Fourier transform, and the impulse responses are not sufficiently time-limited; thus time-domain aliasing is introduced as per the sampling theorem. This prohibits reliable estimation of, for example, the $99.99 \%$ energy breakpoint. The 99\% energy breakpoint, on the other hand, is at least 18 times lower than the period of the aliased impulse response, and thus can be reliably estimated.

\section{CONCLUSIONS}

Simple parametric crosstalk models are useful tools in VDSL system engineering. The evolution towards FTTC/FTTB architectures implies shorter twisted pair segments, and correspondingly wider usable system bandwidth. This brings up the issue of whether or not existing models for NEXT and FEXT are valid in the FTTC/FTTB scenario.

An extensive measurement campaign was undertaken in order to address this question. An important conclusion of the ensuing analysis is that the simple log-normal statistical models in [3] capture the essential aspects of both NEXT and FEXT over the extended range of frequencies considered. Intuition regarding the behavior of FEXT for the shortest loops has been confirmed by analysis. A number of useful fitted model parameters were also provided.

\section{ACKNOWLEDGMENTS}

The authors would like to thank the anonymous reviewers for their insightful comments. This work was supported by the EU-FP6 under U-BROAD STREP contract 506790.

\section{REFERENCES}

[1] "Spectrum Management for Loop Transmission Systems," ANSI Standard T1.417-2003, Section A.3.2.1. 
[2] E. Karipidis, N. Sidiropoulos, A. Leshem, and L. Youming, "Experimental evaluation of capacity statistics for short VDSL loops," IEEE Transactions on Communications, vol. 53, no. 7, pp. 1119-1122, 2005.

[3] J.-J. Werner, "The HDSL environment [high bit rate digital subscriber line]," IEEE Journal on Selected Areas in Communications, vol. 9, no. 6, pp. 785-800, 1991.

[4] K. Kerpez, "Models for the numbers of NEXT disturbers and NEXT loss," Contribution number T1E1.4/99-471, October 1999, available at http://contributions.atis.org/UPLOAD/NIPP/ NAI/9E144710.pdf.

[5] A. Leshem, "Multichannel noise models for DSL I: Near end crosstalk," Contribution T1E1.4/2001-227, September 2001, available at http://contributions.atis.org/UPLOAD/NIPP/NAI/ 1E142270.zip.

[6] S. H. Lin, "Statistical behaviour of multipair crosstalk," Bell System Technical Journal, vol. 59, no. 6, pp. 955-974, 1980.

[7] Norme Française \# NF C 93-527-2, July 1991.

E. Karipidis received the Diploma in electrical and computer engineering from the Aristotle University of Thessaloniki, Greece, and the M.S. degree in communications engineering from the Technical University of Munich, in 2001 and 2003, respectively. He worked as an intern from February 2002 to October 2002 in Siemens ICM, and from December 2002 to November 2003 in the Wireless Solutions Lab of DoCoMo Euro-

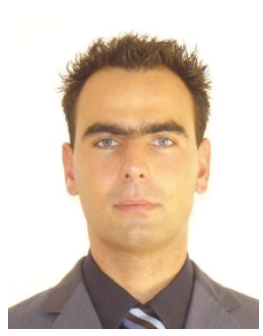
Labs, both in Munich, Germany. He is currently a Ph.D. candidate in the Telecommunications Division, Department of Electronic and Computer Engineering, Technical University of Crete, Chania, Greece. His broad research interests are in the area of signal processing for communications, with current emphasis on MIMO VDSL systems, convex optimization, and applications in transmit precoding for wireline and wireless systems. He is Member of the Technical Chamber of Greece and Student Member of the IEEE.

N. Sidiropoulos received the Diploma in electrical engineering from the Aristotle University of Thessaloniki, Greece, and M.S. and Ph.D. degrees in electrical engineering from the University of Maryland at College Park (UMCP), in 1988, 1990, and 1992, respectively. He has been an Assistant Professor in the Department of Electrical Engineering, University of Virginia (19971999), and Associate Professor in the De-

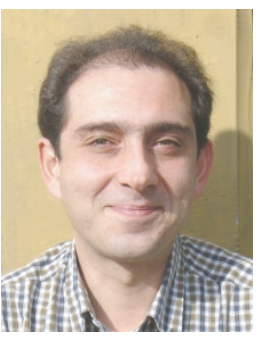
partment of Electrical and Computer Engineering, University of Minnesota, Minneapolis (2000-2002). Since 2002, he is a professor in the Department of Electronic and Computer Engineering, Technical University of Crete, Chania, Crete, Greece. His current research interests are in signal processing for communications, and multiway analysis. He is Vice-Chair of the Signal Processing for Communications Technical Committee (SPCOM-TC), and Member of the Sensor Array and Multichannel Processing Technical Committee (SAMTC) of the IEEE SP Society, and Associate Editor for IEEE Transactions on Signal Processing (2000-). He received the U.S. NSF/CAREER Award in June 1998, and an IEEE Signal Processing Society Best Paper Award in 2001. He is an active consultant for industry in the areas of frequency hopping systems and signal processing for xDSL modems.

A. Leshem received the B.S. degree (cum laude) in mathematics and physics, the M.S. degree (cum laude) in mathematics, and the Ph.D. degree in mathematics all from the Hebrew University, Jerusalem, Israel, in 1986, 1990, and 1998, respectively. From 1998 to 2000, he was with Faculty of Information Technology and Systems, Delft University of Technology, the Netherlands, as a postdoctoral fellow working on algorithms for reduction of terrestrial electromagnetic interference in radioastronomical radio-telescope antenna arrays and signal processing for communication. From 2000 to 2003, he was Director of advanced technologies with Metalink Broadband. He was responsible for research and development of new DSL and wireless MIMO modem technologies. From 2000 to 2002, he was also a Visiting Researcher at Delft University of Technology. Since October 2002, he has been a Senior Lecturer in the new School of Electrical and Computer Engineering, at Bar-Ilan University. From 2003 to 2005, he also was Technical Manager of the U-BROAD consortium developing technologies to provide $100 \mathrm{Mbps}$ and beyond over copper lines. His main research interests include transmission over copper lines including multiuser and multichannel transmission techniques, array and statistical signal processing with applications to multiple-element sensor arrays in radio-astronomy and wireless communications, radio-astronomical imaging methods, set theory, logic and foundations of mathematics.

Li Youming received the B.S. degree in computational mathematics from Lan Zhou University, Lan Zhou, China, in 1985, the M.S. degree in computational mathematics from Xi'an Jiaotong University in 1988, and the Ph.D. degree in electrical engineering from Xi Dian University. From 1988 to 1998 , he worked in the Department of Applied Mathematics, Xidian University, where he was an Associate Professor. From

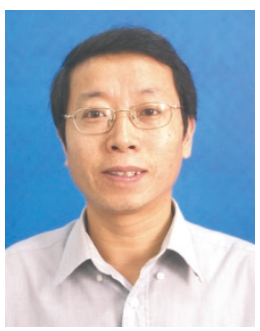
1999 to 2000, he was a Research Fellow in the School of EEE, Nanyang Technological University. From 2001 to 2003, he joined DSO National Laboratories, Singapore. From 2001 to 2004, he was a postdoctoral research fellow in School of Engineering, BarIlan University, Israel. He is now working in the Faculty of Information Science and Engineering, Ningbo University. His research interests are in the areas of statistical signal processing and its application in wireline and wireless communications and radar.

R. Tarafi was born on October 20, 1968. $\mathrm{He}$ is an Engineer at the Ecole Nationale d'Ingénieurs de Brest (ENIB). In 1998, he received the title of Docteur of the University of Brest. He joined the National Research Center of France Telecom in 1998, where he is in charge of modelization and investigation studies related to the EMC of the France Telecom telecommunication network.

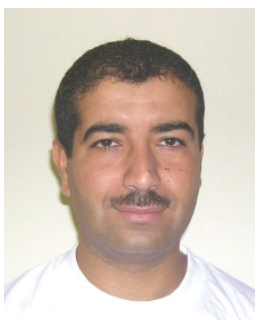


M. Ouzzif received the Engineering degree as well as the M.S. degree in electrical engineering from INSA (Institut National des Sciences Appliquées) of Rennes in 2000 and the Ph.D. degree in electronics from INSA in 2004. Since November 2000, she has been with FranceTelecom R\&D. Her current interests include multiuser transmissions and their application to wireline communications.

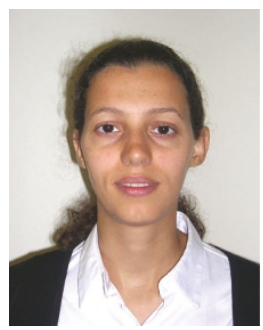

